\title{
Determination of the refractive index and thickness of holographic silver halide materials by use of polarized reflectances
}

\author{
Augusto Beléndez, Tarsicio Beléndez, Cristian Neipp, and Inmaculada Pascual
}

\begin{abstract}
A method to determine the refractive index and thickness of silver halide emulsions used in holography is presented. The emulsions are in the form of a layer of film deposited on a thick glass plate. The experimental reflectances of $p$-polarized light are measured as a function of the incident angles, and the values of refractive index, thickness, and extinction coefficient of the emulsion are obtained by using the theoretical equation for reflectance. As examples, five commercial holographic silver halide emulsions are analyzed. The procedure to obtain the measurements and the numerical analysis of the experimental data are simple, and agreement of the calculated reflectances, by use of the thickness and refractive index obtained, with the measured reflectances is satisfactory. (C) 2002 Optical Society of America

OCIS codes: $120.4530,120.5700,090.2900,310.6860,090.0090$.
\end{abstract}

\section{Introduction}

Silver halide emulsion is the most popular material for hologram recording because of its relatively high sensitivity, spectral sensitivity matched to a range of lasers, low cost, and easy handling.1,2 The emulsions analyzed consist of fine grain silver halide crystals (basically, silver bromide) suspended in a gelatin and deposited on a thick glass plate. Determination of the refractive index and thickness is one of the first steps in the characterization of a holographic recording material, as well as in certain applications, such as the manufacture of holographic optical elements. The refractive index of pure gelatin is approximately 1.52 to 1.54 for the visible spectrum, and the refractive index of the silver bromide crystals is approximately 2.25, and the refractive index of a typical

\footnotetext{
A. Beléndez (augusto@disc.ua.es) and C. Neipp are with the Departamento de Física, Ingeniería de Sistemas y Teoría de la Señal, Universidad de Alicante, Apartado 99, E-03080 Alicante, Spain. T. Beléndez is with Departamento de Ciencia y Tecnología de los Materiales, Universidad Miguel Hernández, Avda. del Ferrocarril, s/n, E-03202, Elche, Spain. I. Pascual is with the Departamento Interuniversitario de Óptica, Universidad de Alicante, Apartado 99, E-03080 Alicante, Spain.

Received 22 April 2002; revised manuscript received 7 August 2002.

0003-6935/02/326802-07\$15.00/0

(C) 2002 Optical Society of America
}

silver halide emulsion for holographic applications is $\sim 1.6$. $^{1}$

Five commercial silver halide emulsions commonly used in holography have been considered in this paper. Two of them (PFG-01 and PFG-03M) are manufactured by the Slavich company in Russia. Two of them (BB-640 and BB-520) were initially manufactured by Holographic Recording Technologies in Germany, even though they are now being manufactured by another company, Colour Holographics, in England. Finally, the fifth emulsion (Agfa 8E75 HD) was manufactured by the Agfa company in Belgium. Although production of this last emulsion was discontinued at the end of 1997, it was one of the emulsions most frequently used for holographic recording for more than 25 years, and is still an emulsion of reference, mainly for comparing the results obtained by using the new emulsions with those achieved previously with the Agfa plates. The mean silver-halide grain size is about $10 \mathrm{~nm}$ for PFG-03M, $20 \mathrm{~nm}$ for BB-640 and BB-520, and 40 nm for PFG-01 and Agfa 8E75 HD emulsions. ${ }^{1-5}$

There are several methods for measuring the complex refractive index and thickness of thin films, e.g., angle-of-reflectance versus angle-of-incidence methods or versus the wavelength, ${ }^{6-11}$ ellipsometry, ${ }^{12,13}$ polarization-independent reflectance matching, ${ }^{14,15}$ or heterodyne interferometry. ${ }^{16}$ In these methods, the reflectances of $s$ and/or $p$ polarization at several 


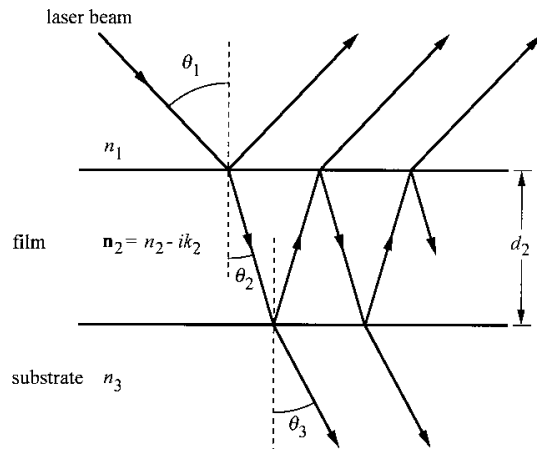

Fig. 1. Reflections from a single layer of absorbing material on a transparent substrate.

different angles of incidence, polarization conditions, or phase differences must be measured.

In this paper a simple method for measuring the refractive index and thickness of silver halide emulsions used in holography is proposed. The technique is based on the film resonance method used by Tholl et $a l .{ }^{17}$ for dichromated gelatin holographic films. However, the method we use has important differences with respect to that of Tholl et al., especially in relation to the analysis of the experimental data and the theoretical fitting. The procedure used here is based on analysis of the interference phenomena in the reflected light produced by the emulsion layer. With $p$-polarized light, the reflectance at different incident angles is measured and the simultaneous determination of the optical constants and thickness can be achieved by use of a computer program.

\section{Theoretical Analysis}

The thin-film structure considered here is shown in Fig. 1. A single layer of absorbing material with complex refractive index $n_{2}=n_{2}-i k_{2}$, where $n_{2}$ is the refractive index and $k_{2}$ is the extinction coefficient, and thickness $d_{2}$ is located between two semiinfinite regions of nonabsorbing materials with refractive indexes $n_{1}$ and $n_{3}$, respectively. Region 1 receives the name of incident medium, region 2 is the film, and we call the substrate region 3. A laser beam, linearly polarized parallel to the plane of incidence ( $p$ polarization), of wavelength $\lambda$ in region 1 is incident at an angle $\theta_{1}$ on the film. The amplitude reflectance $r$ is given by the following equation ${ }^{8,18}$ :

$$
r=\frac{r_{12}+r_{23} \exp (-2 i \beta)}{1+r_{12} r_{23} \exp (-2 i \beta)}
$$

where $r_{12}$ and $r_{23}$ are the Fresnel amplitude reflection coefficients of the 1-2 and 2-3 single interfaces, and $\beta$ is the phase difference between the waves reflected from the first and second surfaces of the film layer, and can be written as

$$
\beta=\frac{4 \pi}{\lambda} d_{2} \mathbf{n}_{2} \cos \theta_{2}
$$

The amplitude reflection coefficients $r_{12}$ and $r_{23}$ for $p$ polarization can be expressed as

$$
\begin{aligned}
& r_{12}=\frac{\mathbf{n}_{2} \cos \theta_{1}-n_{1} \cos \theta_{2}}{\mathbf{n}_{2} \cos \theta_{1}+n_{1} \cos \theta_{2}}, \\
& r_{23}=\frac{n_{3} \cos \theta_{2}-\mathbf{n}_{2} \cos \theta_{3}}{n_{3} \cos \theta_{2}+\mathbf{n}_{2} \cos \theta_{3}},
\end{aligned}
$$

where $\mathbf{n}_{2}$ and $\theta_{2}$ are complex values. Both $n_{1}, n_{2}$ and $n_{3}$, as well as $k_{2}$, are functions of the wavelength $\lambda$ of the incident beam. Because the film is an absorbing layer, $r_{12}$ and $r_{23}$ are generally complex. Following Ref. 8, we can write:

$$
\mathbf{n}_{2} \cos \theta_{2}=u_{2}-i v_{2},
$$

where $u_{2}$ and $v_{2}$ are real. Using Snell's law we obtain:

$$
\mathbf{n}_{2} \sin \theta_{2}=n_{1} \sin \theta_{1}=n_{3} \sin \theta_{3} .
$$

Substituting Eqs. (5) and (6) in Eqs. (2), (3), and (4) gives us $r_{12}, r_{23}$, and $\beta$ as a function of the incident angle $\theta_{1}$, the refractive indexes $n_{1}, n_{2}$, and $n_{3}$, extinction coefficient $k_{2}$, thickness $d_{2}$, and wavelength $\lambda$ :

$$
\begin{aligned}
& r_{12}=\frac{\left(n_{2}-i k_{2}\right)^{2} \cos \theta_{1}-n_{1}\left(u_{2}+i v_{2}\right)}{\left(n_{2}-i k_{2}\right)^{2} \cos \theta_{1}+n_{1}\left(u_{2}+i v_{2}\right)}, \\
& r_{23}=\frac{n_{3}{ }^{2}\left(u_{2}+i v_{2}\right)-\left(n_{2}-i k_{2}\right)^{2}\left(n_{3}{ }^{2}-n_{1}{ }^{2} \sin ^{2} \theta_{1}\right)^{1 / 2}}{n_{3}{ }^{2}\left(u_{2}+i v_{2}\right)+\left(n_{2}-i k_{2}\right)^{2}\left(n_{3}{ }^{2}-n_{1}{ }^{2} \sin ^{2} \theta_{1}\right)^{1 / 2}},
\end{aligned}
$$

$$
\beta=\frac{2 \pi}{\lambda} d_{2}\left(u_{2}+i v_{2}\right),
$$

where

$$
\begin{aligned}
u_{2}^{2}= & \frac{1}{2}\left\{\left(n_{2}{ }^{2}-k_{2}{ }^{2}-n_{1}{ }^{2} \sin ^{2} \theta_{1}\right)+\left[\left(n_{2}{ }^{2}-k_{2}{ }^{2}\right.\right.\right. \\
& \left.\left.\left.-n_{1}{ }^{2} \sin ^{2} \theta_{1}\right)^{2}+4 n_{2}{ }^{2}{k_{2}}^{2}\right]^{1 / 2}\right\}, \\
v_{2}{ }^{2}= & \frac{1}{2}\left\{-\left(n_{2}{ }^{2}-k_{2}{ }^{2}-{n_{1}}^{2} \sin ^{2} \theta_{1}\right)+\left[\left(n_{2}{ }^{2}-k_{2}{ }^{2}\right.\right.\right. \\
& \left.\left.\left.-n_{1}{ }^{2} \sin ^{2} \theta_{1}\right)^{2}+4 n_{2}{ }^{2}{k_{2}}^{2}\right]^{1 / 2}\right\} .
\end{aligned}
$$

From Eqs. (7)-(11) we can see that $r_{12}$ and $r_{23}$, and obviously $r$, vary with the angle of incidence $\theta_{1}$.

The reflectance $R$ can be obtained from Eq. (1) as

$$
R=|r|^{2},
$$

and substituting Eqs. (7), (8), and (9) in Eq. (1), and using Eq. (12) we obtain $R$ as the following function:

$$
R=f\left(n_{1}, n_{2}, n_{3}, k_{2}, d_{2}, \theta_{1}, \lambda\right) .
$$

We suppose that the wavelength $\lambda$ and refractive indexes $n_{1}$ and $n_{3}$ of the incident medium and the substrate, respectively, are known. On the other hand, we experimentally measure the reflectance $R$ as a function of the incident angle $\theta_{1}$. Then there 


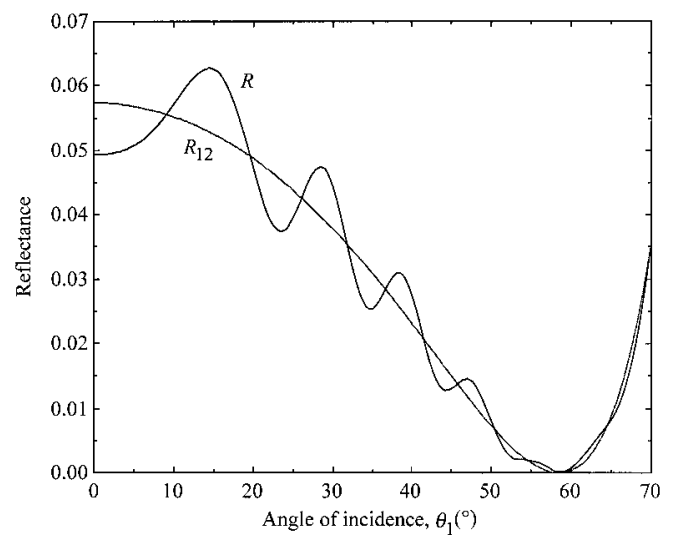

Fig. 2. Calculated reflectance curves $R$ and $R_{12}$ as a function of the angle of incidence for $n_{1}=1, n_{2}=1.63, k_{2}=0.0035, n_{3}=1.52$, $d=6 \mu \mathrm{m}$, and $\lambda=633 \mathrm{~nm}$.

are three unknown parameters, the refractive index $n_{2}$, extinction coefficient $k_{2}$ and thickness $d_{2}$ of the layer. We obtain these parameters by comparing the experimentally measured reflectances $R_{\text {exp }}\left(\theta_{1 j}\right)$, where $j=1,2, \ldots, J$, and $J$ is the number of angles of incidence in medium 1 , with the calculated reflectances $R\left(n_{2}, k_{2}, d_{2}, \theta_{1 j}\right)$. We obtain the values of the optical constants $n_{2}$ and $k_{2}$ for the wavelength $\lambda$ and thickness $d_{2}$ for which the sum of the root mean square $\chi^{2}$ is minimum, where $\chi^{2}$ is given by the following equation:

$$
\chi^{2}=\frac{1}{J^{2}} \sum_{j=1}^{J}\left[R\left(n_{2}, k_{2}, d_{2}, \theta_{1 j}\right)-R_{\exp }\left(\theta_{1 j}\right)\right]^{2} .
$$

\section{Single Layer of Weakly Absorbing Material}

When the thin film is a weakly absorbing medium, such as a silver halide emulsion, then $k_{2} \ll n_{2}$, and from Eqs. (1) and (12), it is easy to see that reflectance $R$ practically oscillates around the reflectance $R_{12}$ owing to the light reflected by the 1-2 interface. Taking into account that $k_{2} \ll n_{2}$, and Eqs. (7) and (10), we can calculate $R_{12}$ approximately as follows:

$$
R_{12} \approx\left[\frac{n_{2} \cos \theta_{1}-\left(n_{2}^{2}-n_{1}^{2} \sin ^{2} \theta_{1}\right)^{1 / 2}}{n_{2} \cos \theta_{1}+\left(n_{2}^{2}-n_{1}^{2} \sin ^{2} \theta_{1}\right)^{1 / 2}}\right]^{2} .
$$

Figure 2 shows the reflectances $R$ and $R_{12}$ evaluated using Eqs. (12) and (15), respectively, as a function of $\theta_{1}$ for a situation in which $k_{2} \ll n_{2}$. For computation we have considered the parameters $n_{1}=$ 1 (air), $n_{3}=1.52$ (glass), $\lambda=633 \mathrm{~nm}$, and for thin film the parameters of a typical photographic emulsion ${ }^{1}$ : $n_{2}=1.63, k_{2}=0.0035$, and $d_{2}=6 \mu \mathrm{m}$. In Fig. 3 we have plotted the difference between $R$ and $R_{12}$ as a function of $\theta_{1}$ for the same numerical example.

As can be seen from Figs. 2 and $3, R$ practically oscillates around $R_{12}$. For this reason, and to minimize Eq. (14), first we determine an approximate value of the refractive index $n_{2}$ by fitting the reflectivity $R_{12}$ [given by Eq. (15)] to the experimental data. Then a computer program immediately uses this value to begin the minimization process of $\chi^{2}$ to ob-

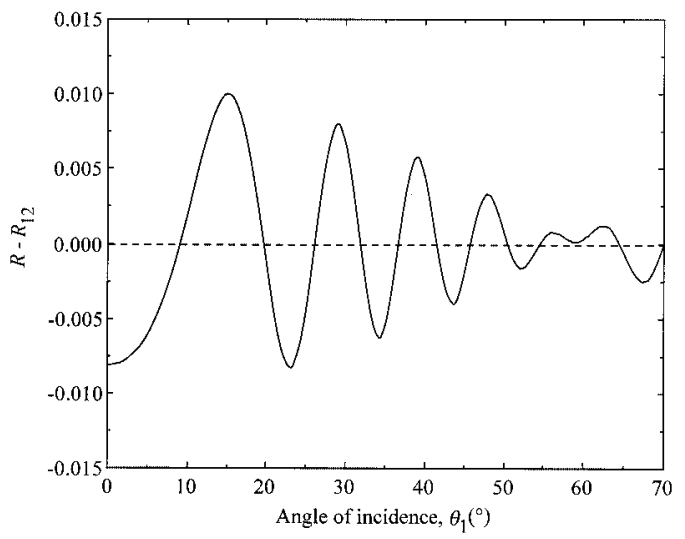

Fig. 3. Difference between $R$ and $R_{12}$ as a function of the angle of incidence for the numerical example considered in Fig. 2.

tain the optical constants $n_{2}$ and $k_{2}$ for $\lambda$ and the thickness $d_{2}$ of the layer under analysis.

\section{Experimental Procedure}

To obtain the values of reflectivity as a function of the incident angle, the experimental setup shown in Figure 4 was considered. The light from a He-Ne laser is divided into two by means of a beam splitter. One of the beams impinges on the photographic plate, whereas the other beam impinges on an optical power meter, F1, to record information about the fluctuations in the laser power during the measurements. A mirror is mounted on the photographic plate perpendicular to it. In this way, the direction of the beam reflected by the set formed by the plate and the mirror is parallel to the incident beam direction. Therefore the direction of the reflected beam is independent of the angle formed by the incident beam with the normal to the plate. The light intensity reflected by the holographic plate/mirror system was measured by means of an optical power meter, F2, placed in the focal plane of a collimating lens. Both F1 and F2 were picowatt digital optical power meters with low power silicon detectors.

The plate was mounted on a high-precision motorized rotation stage that was controlled electronically

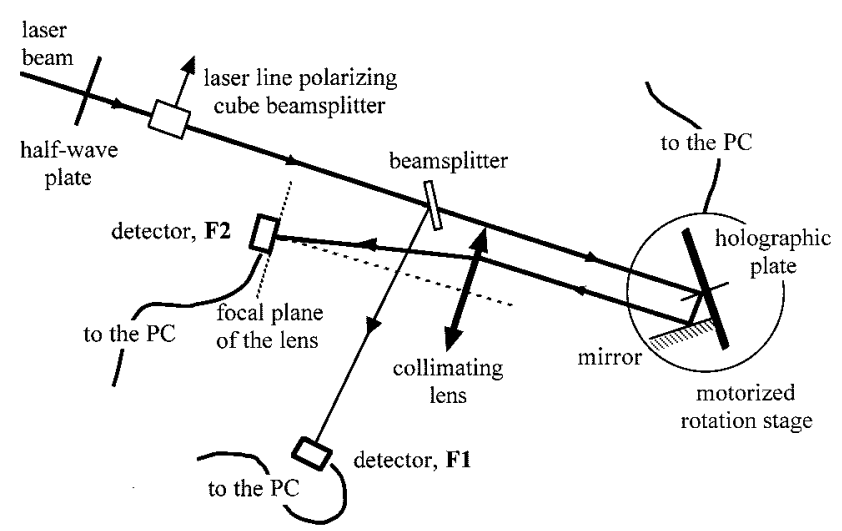

Fig. 4. Schematic diagram of the experimental setup. 


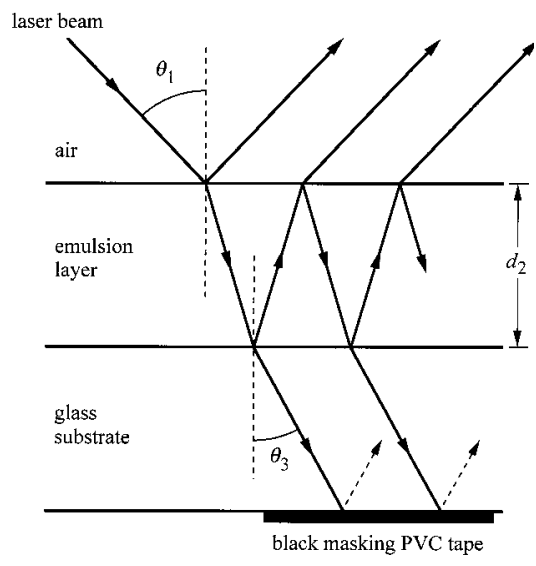

Fig. 5. Experimental procedure used to eliminate the backreflected light produced at the glass-air interface at the rear side of the holographic plates.

using a point-to-point motion controller connected to a personal computer across an IEEE-488 interface. The rotating device had an angular resolution of $0.001^{\circ}$. The plate was illuminated with a beam polarized parallel to the plane of incidence from an $\mathrm{He}-\mathrm{Ne}$ laser polarized perpendicular to the plane of incidence. To obtain a laser beam polarized parallel to the incident plane, the laser beam first went through a half-wave plate whose slow axis formed an angle of $45^{\circ}$ with the polarization direction of the laser beam. Afterwards, the beam went through a laser line polarizing cube beam-splitter from Newport for the wavelength $632.8 \mathrm{~nm}$. The $p$-polarized light was transmitted, while the $s$-polarized light was reflected. Its extinction ratio was less than $10^{-3}$. This cube beam splitter was manufactured from BK7, grade A, fine annealed optical glass and was placed and positioned on a prism table. The reflected intensity was measured varying the angle of incidence between $25^{\circ}$ and $55^{\circ}$ at intervals of $0.25^{\circ}$.

To efficiently eliminate the back-reflected light produced at the glass-air interface at the rear side of the holographic plate a black self-adhesive poly vinyl chloride (PVC) masking tape was stuck onto the back surface of the plane parallel glass plate (Fig. 5).19

The losses of the different optical components of the measurement system were taken into account in the data analysis. However, as in Ref. 17, instead of measuring the losses for each optical component as a function of the incident angle, the experimental setup was calibrated with a parallel glass window. This parallel window had a clear aperture $50.8 \mathrm{~mm}$ in diameter and $10 \mathrm{~mm}$ thick, with a wave-front distortion less than $\lambda / 10$ at $632.8 \mathrm{~nm}$ over the clear aperture. The glass window was manufactured by Newport from BK7, grade A, fine annealed optical glass with a refractive index of $n=1.5151$ at a wavelength of $632.8 \mathrm{~nm}$. To calibrate the system the parallel window was placed at the same place on the holographic plate, and the reflected light was measured by varying the angle of incidence, $\theta_{1}$, between $25^{\circ}$ and $55^{\circ}$ at intervals of $0.25^{\circ}$. To eliminate the

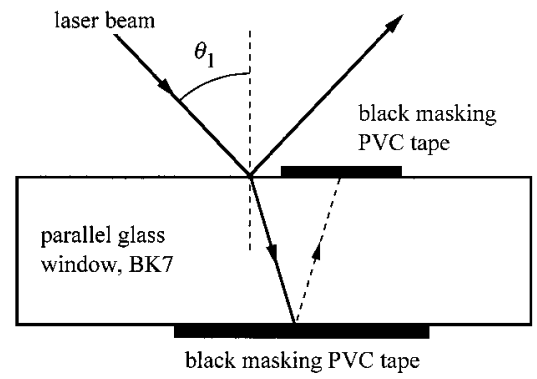

Fig. 6. Experimental procedure to eliminate the reflection produced at the glass-air interface of the parallel glass window.

reflection produced at the air-glass and glass-air interfaces, black self-adhesive PVC masking tape was stuck onto the parallel glass plate as can be seen in Fig. 6. In this way, light is only reflected at the first air-glass interface of the plate. Therefore it is possible to consider only a single beam reflected by the air-glass interface with a refractive index of 1.5151 , and the following theoretical equation for reflectivity can be used:

$$
R_{\mathrm{BK} 7}=r_{\mathrm{ag}}^{2},
$$

where $r_{\text {ag }}$ is the Fresnel amplitude reflection coefficient of the single air-glass interface. $R^{\prime}$ was calculated, as a function of the angle of incidence $\theta_{1}$, as the quotient of the reflected light, $I_{r}$, impinging onto the optical power meter F2, and the incident light, $I_{i}$, impinging onto the optical power meter F1:

$$
R_{\mathrm{BK} 7}{ }^{\prime}\left(\theta_{1}\right)=\frac{I_{r}\left(\theta_{1}\right)}{I_{i}\left(\theta_{1}\right)} .
$$

The ratio between the theoretical reflectivity for the $\mathrm{BK} 7$ glass plate, $R_{\mathrm{BK} 7}$, and the experimentally measured reflectivity, $R_{\mathrm{BK} 7}$, gives the calibration factor for the incident angle, $\theta_{1}$ :

$$
f\left(\theta_{1}\right)=\frac{R_{\mathrm{BK} 7}\left(\theta_{1}\right)}{R_{\mathrm{BK} 7}{ }^{\prime}\left(\theta_{1}\right)}
$$

To obtain the experimental reflectances, $R_{\text {exp }}\left(\theta_{1}\right)$, for each angle of incidence, the values of the reflectance, $R^{\prime}\left(\theta_{1}\right)$, obtained for each holographic plate are multiplied by the factor $f\left(\theta_{1}\right)$ :

$$
R_{\exp }\left(\theta_{1}\right)=f\left(\theta_{1}\right) R^{\prime}\left(\theta_{1}\right) .
$$

The fitting of the theoretical reflectivity [Eq. (13)] to the experimental data (with $J=121$ measurements of the incident angle $\theta_{1}$ ) of the reflectance for the analyzed holographic plates is carried out, as explained before, minimizing the quantity $\chi^{2}[\mathrm{Eq}$. (14)], which we write as

$$
\chi^{2}=\frac{1}{J^{2}} \sum_{j=1}^{J}\left(R_{j}-R_{\exp , j}\right)^{2},
$$

where $R_{\exp , j}=R_{\exp }\left(\theta_{j}\right)$ and $R_{j}=R\left(n_{2}, k_{2}, d_{2}, \theta_{1 j}\right)$ is of the calculated reflectance.

To carry out the fitting of the theoretical reflectivi- 


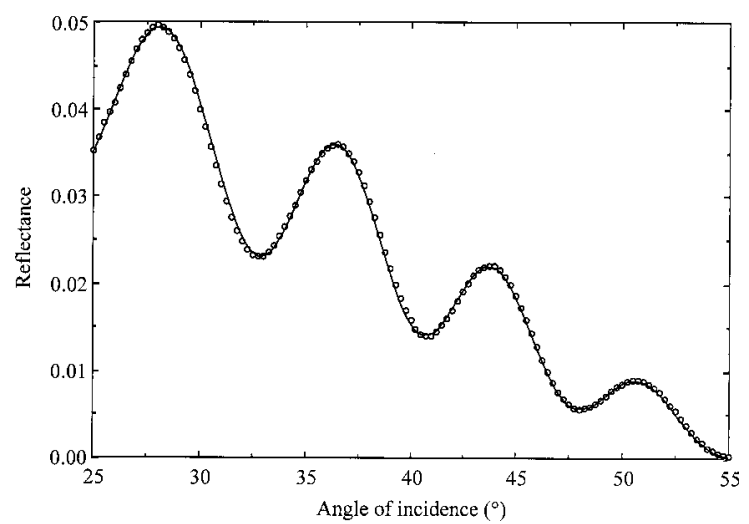

Fig. 7. Measured (open circles) and calculated (continuous wave) reflectances for PFG-01 emulsion while varying the angle of incidence from $25^{\circ}$ to $55^{\circ}$. Light polarized parallel to the plane of incidence.

ties to the experimental data, it is necessary to know the refractive index $n_{3}$ of the glass substrate, assuming that $k_{3}=0$ (for glass substrates the extinction coefficient takes values of less than $1.6 \times 10^{-6}$ between $400 \mathrm{~nm}$ and $700 \mathrm{~nm}^{20}$ ). We took a plate of each of the different emulsions, Agfa, BB and PFG, ensured that the emulsions were completely removed, and the glass plates were immersed in a chromic mixture solution for $24 \mathrm{~h}$. The plates were then rinsed in running water and dried. Afterwards, black self-adhesive PVC masking tape was stuck on the glass plates, and we measure the reflectance for a plane wave with its electric vector parallel to the plane of incidence. A least square fit was made to fit the theoretical function $R_{12}$ of Eq. (15) to the experimental data (once these had been corrected taking into account the calibration $f$ of the measuring system) considering that now $n_{1}=1$ (air) and $n_{2}$ is the refractive index of the glass substrate, and we obtain the refractive indexes of the glass substrates for Agfa, $\mathrm{BB}$, and PFG plates by minimizing Eq. (20). The values obtained for the refractive indexes of the glass substrates were $1.5152 \pm 0.0012,1.5148 \pm 0.0011$,

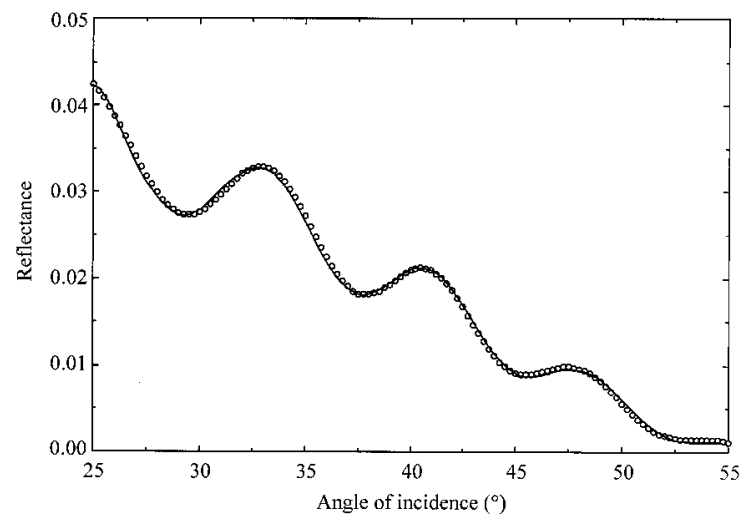

Fig. 8. Measured (open circles) and calculated (continuous wave) reflectances for PFG-03M emulsion while varying the angle of incidence from $25^{\circ}$ to $55^{\circ}$. Light polarized parallel to the plane of incidence.

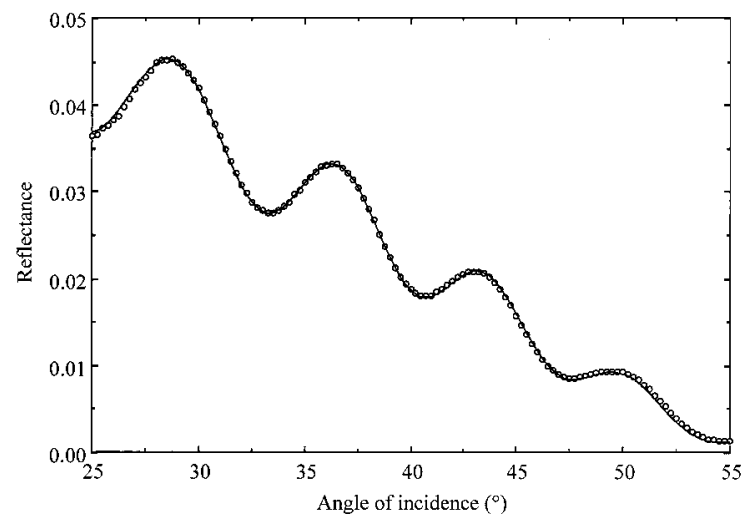

Fig. 9. Measured (open circles) and calculated (continuous wave) reflectances for BB-640 emulsion while varying the angle of incidence from $25^{\circ}$ to $55^{\circ}$. Light polarized parallel to the plane of incidence.

and $1.5067 \pm 0.0010$, for Agfa, BB, and PFG plates, respectively.

\section{Experimental Results}

As we pointed out in Section 1, five red silver halide emulsions used in holography were studied: PFG01, PFG-03M, BB-640, BB-520, and Agfa 8E75 HD. Figures 9 through 11 show the reflectivity as a function of the angle of incidence for the holographic plates considered. The dots correspond to the experimental data, and the continuous lines correspond to the reflectances calculated with the values obtained of the refractive index, extinction coefficient, and thickness of the film. Comparing the experimentally measured reflectances with the calculated curves, we can see that the agreement is satisfactory. Table 1 shows the results obtained for the refractive index, extinction coefficient, and thickness of the five emulsions analyzed, together with the corresponding value of $\chi^{2}$. As can be seen, $\chi^{2}$ varies from $3.24 \times$ $10^{-10}$ to $11.89 \times 10^{-10}$, which means that $\chi$ varies from 0.000018 to 0.000034 . These last values should be the average difference between the exper-

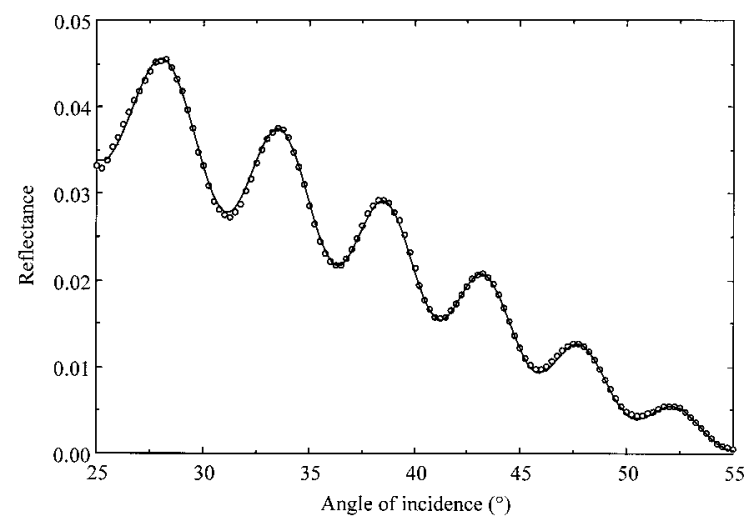

Fig. 10. Measured (open circles) and calculated (continuous wave) reflectances for BB-520 emulsion while varying the angle of incidence from $25^{\circ}$ to $55^{\circ}$. Light polarized parallel to the plane of incidence. 


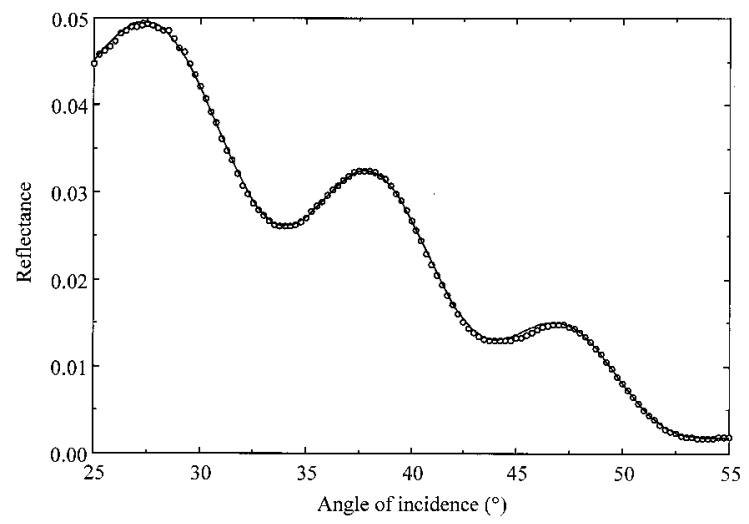

Fig. 11. Measured (open circles) and calculated (continuous wave) reflectances for Agfa $8 \mathrm{E} 75 \mathrm{HD}$ emulsion while varying the angle of incidence from $25^{\circ}$ to $55^{\circ}$. Light polarized parallel to the plane of incidence.

imental reflectances and those calculated theoretically from the fitting parameters. It was found that when carrying out the theoretical fittings, the values of $n_{2}$ and $d_{2}$ obtained did not change appreciably, although a value of $n_{3}=1.51$ was taken in each case for the refractive index of the glass substrate. This fact indicates that it is not necessary to have a precise knowledge of $n_{3}$ to determine $n_{2}$ and $d_{2}$. Nevertheless, to determine $k_{2}$ with a certain precision it is necessary to have a precise value of $n_{3}$.

\section{Conclusions}

A method that enables us to obtain the refractive index, extinction coefficient, and thickness of silver halide emulsions used in holography has been developed. The agreement of the reflectances calculated by use of the optical constants and thickness obtained by this method with the measured reflectances is satisfactory and confirms the applicability of the method to holographic plates. This study of reflectance demonstrates that the procedure for elimination of the reflections that occur at the glass-air interface of holographic plates gives rise to optimum results and has the advantage that its practical application is straightforward compared to other methods involving the use of matching liquids - which are often dangerous or toxic-and a prism attached to the substrate. ${ }^{17}$ The technique has several merits, such as the use of a simple optical setup, easy operation, and high accuracy of measurement. The method is applicable to other holographic recording materials, such as photopolymers, dichromated gelatins, or pho- toresists, provided these materials are deposited on glass and the thickness of the layer is not very great.

This work was partially financed by the Comisión Interministerial de Ciencia y Tecnología, Spain) under project No. MAT2000-1361-C04-04 and the Oficina de Ciencia y Tecnología (Generalitat Valenciana, Spain) under project No. GV01-130.

\section{References}

1. H. I. Bjelkhagen, Silver Halide Recording Materials for Holography and Their Processing (Springer-Verlag, Berlin, 1993).

2. C. Neipp, I. Pascual, and A. Beléndez, "Recent advances in new holographic silver halide materials," in Recent Research Developments in Optics, Vol. I (Research Signpost, Trivandrum, India, 2001), pp. 35-56.

3. C. Neipp, I. Pascual, and A. Beléndez, "Theoretical and experimental analysis of overmodulation effects in volume holograms recorded on BB-640 emulsion," J. Opt. A. Pure Appl. Opt. 3, 504-513 (2001).

4. C. Neipp, I. Pascual, and A. Beléndez, "Bleached silver halide volume holograms recorded on Slavich PFG-01 emulsion: The influence of the developer," J. Mod. Opt. 48, 1479-1494 (2001).

5. S. J. Zacharovas, D. B. Ratcliffe, G. R. Skokov, S. P. Vorobyov, P. I. Kumonko, and Y. A. Sazonov, "Recent advances in holographic materials from Slavich," in Holography 2000, T. H. Jeong and W. K. Sobotka, Proc. SPIE 4149, 73-80 (2000).

6. E. D. Palik, Handbook of Optical Constants of Solids (Academic, New York, 1985), pp. 69-87.

7. A. A. Dakhel, "Optical constants of evaporated gadolinium oxide," Pure Appl. Opt. 3, 452-454 (2001).

8. T. Kihara and K. Yokomori, "Simultaneous measurement of refractive index and thickness of thin film by polarized reflectances," Appl. Opt. 29, 5069-5073 (1989).

9. J. J. Ruíz-Pérez, J. M. González-Leal, D. A. Minkov, and E. Márquez, "Method for determining the optical constants of thin dielectric films with variable thickness using only their shrunk reflection spectra," J. Phys. D.: Appl. Phys. 34, 24892496 (2001).

10. R. Z. Vitlina and G. I. Surdotovich, "A 'blurred film' model inpolarized light reflectometry for characterization of thick films and surface layers," J. Phys. D. Appl. Phys. 34, 25932598 (2001).

11. C. Razzetti, S. Romani, F. Bissoli, M. Zha, and L. Zanotti, "Determination of the refractive indices by variable azimuth reflectometry from a single sample face," J. Opt. A. Pure Appl. Opt. 3, 108-113 (2001).

12. J. Lekner, "Determination of complex refractive index and thickness of a homogeneous layer by combined reflection and transmission ellipsometry," J. Opt. Soc. Am. A 11, 2156-2158 (1994).

13. R. M. A. Azzam and N. M. Bashara, Ellipsometry and Polarized Light (North-Holland, Amsterdam, 1989).

Table 1. Best Fitting Parameters for the Optical Constants and the Thickness

\begin{tabular}{lcrrr}
\hline \multicolumn{1}{c}{ Emulsion } & \multicolumn{1}{c}{$n_{2}$} & \multicolumn{1}{c}{$d_{2}(\mu \mathrm{m})$} & $k_{2}$ & $\chi^{2}$ \\
\hline PFG-01 & $1.609 \pm 0.002$ & $7.31 \pm 0.01$ & $0.00008 \pm 0.00006$ & $11.892 \times 10^{-10}$ \\
PFG-03M & $1.569 \pm 0.002$ & $7.00 \pm 0.01$ & $0.00099 \pm 0.00011$ & $7.709 \times 10^{-10}$ \\
BB-640 & $1.624 \pm 0.002$ & $7.86 \pm 0.01$ & $0.00382 \pm 0.00007$ & $4.634 \times 10^{-10}$ \\
BB-520 & $1.609 \pm 0.002$ & $11.42 \pm 0.01$ & $0.00138 \pm 0.00006$ & $7.521 \times 10^{-10}$ \\
Agfa 8E75 HD & $1.632 \pm 0.002$ & $5.77 \pm 0.01$ & $0.00377 \pm 0.00009$ & $3.237 \times 10^{-10}$ \\
\hline
\end{tabular}


14. R. M. A. Azzam, "Polarization-independent reflectance matching (PIRM). A technique for the determination of the refractive index and thickness of the transparent films," J. Opt. 8, 201-205 (1977).

15. Y. Cui and R. M. A. Azzam, "Determination of the refractive index and thickness of transparent pellicles by use of the polarization-independent absentee-layer condition," Appl. Opt. 35, 5040-5043 (1996).

16. M-H. Chiu, J-Y. Lee, and D-C Su, "Complex refractive-index measurement based on Fresnel's equations and the uses of heterodyne interferometry," Appl. Opt. 38, 4047-4052 (1999).

17. H. D. Thöll, M. Dömen, and C. D. Stojanoff, "Determination of the mean refractive index and the thickness of dichromated gelatin holographic films using the thin film resonance method," in Holographic Materials, T. J. Trout, ed., Proc. SPIE 2405, 76-87 (1997).

18. M. Born and E. Wolf, Principles of Optics (Pergamon, Oxford, 1993).

19. A. Beléndez, C. Neipp, and I. Pascual, "Analysis and elimination of boundary reflections in transmission holograms," Opt. Laser Technol. 30, 555-556 (1999).

20. R. J. Martín-Palma, J. M. Martínez-Duart, and A. Malats, "Determination of the optical constants of thin oxide and silver thick fims for their use in multilayer low-emissivity coatings," Bol. R. Soc. Esp. Cerám. Vidrio 39, 472-475 (2000). 